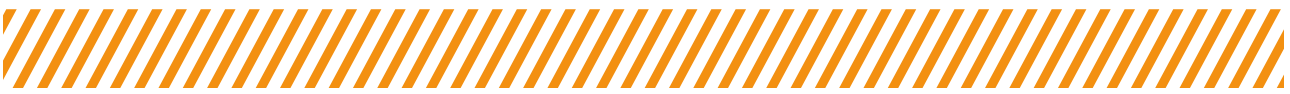

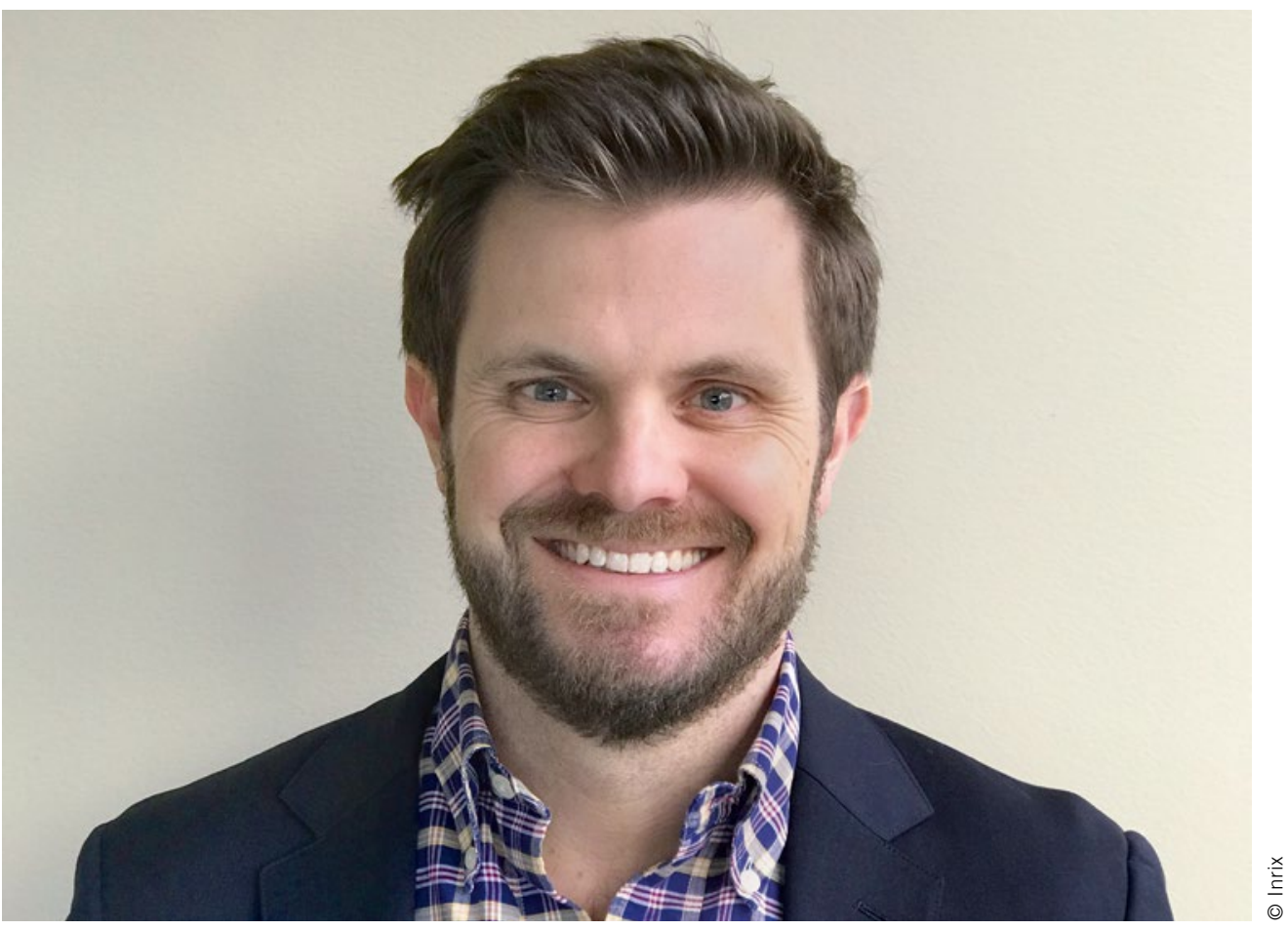

Avery Ash

Autonomous Vehicle

Market Strategist at Inrix

in Washington DC (USA)

\section{Engineers Must Illuminate}

People face disruptive technological changes in many areas of life - at work, in their leisure time and, increasingly, in their mobility decisions. Electromobility along with connected and highly automated driving are front and center, particularly in the context of a promised future that is safer, more convenient and more efficient. However, surveys continue to show that the public is still skeptical about the safety and performance of self-driving vehicles.

This skepticism is not due to failures of the technology itself respondents who have used advanced driver assistance systems overwhelmingly trust and support them - instead this unease is tied to misinformation, a lack of understanding and general reservations about ceding control from a human driver to a vehicle.

As experts in the automotive industry, we must play a central role to build the trust of the general public in the potential for, readiness of and limitations that accompany this new technology. The key resources to build this trust are data and transparency, that will ensure academics, policymakers and consumers can readily measure and confirm the performance of these new vehicles. This means encouraging cities and government stakeholders to pursue data-driven planning for these vehicles and be prepared to measure the impact on key transportation metrics. This also means making engineers and technicians, in addition to marketing and communications profes- sionals, available as credible experts to explain the technology, its benefits and its limitations.

Even if it is initially difficult to communicate a complex technology and disruptive change, one thing facilitates the task: we have many good arguments on our side that will resonate with the public. For example, big data and cloud allow us to offer enhanced safety functions with real-time data, which wasn't possible before, e.g. connected car drivers are already being notified in advance about ice on the road or fog. Drivers are shown the fastest route, based on precise data gathered by their navigation system. You might think that most people use navigation regularly, yet only few use the navigation system on roads that they drive on daily because they know their way around and think they dont't need navigation, as a recent Inirx survey shows. However, using navigation will become increasingly important as it is able to optimally direct traffic and ultimately relieve some routes of heavy traffic. It is incumbent on the manufacturers and operators of highly automated vehicles to highlight these technological opportunities by enabling and encouraging their experts engineers, data scientists and others - to engage with public stake holders to build trust. The successful promotion and adoption of this technology depends on these efforts and they are essential to realising the transformative benefits of these new vehicles. 